\title{
Post-transplant lymphoproliferative disorder presenting as a tumor adjacent to the renal allograft: A case report and review of the literature
}

\author{
CHEN GAO ${ }^{1}$, LONGKAI PENG ${ }^{1}$, FENGHUA PENG $^{1}$, TING TUO ${ }^{1}$ and DAIQIANG LI ${ }^{2}$ \\ Departments of ${ }^{1}$ Urological Organ Transplantation and ${ }^{2}$ Pathology, Second Xiangya Hospital, \\ Central South University, Changsha, Hunan 41011, P.R. China
}

Received February 16, 2014; Accepted September 19, 2014

DOI: $10.3892 / \mathrm{ol} .2014 .2586$

\begin{abstract}
Post-transplant lymphoproliferative disorder (PTLD) is a potentially fatal complication of solid organ transplantation. The current report presents the case of a 42-year-old male who developed PTLD within the first year following renal transplantation. The disorder manifested as a tumor adjacent to the lower pole of the renal allograft and resulted in urinary obstruction. Durable complete remission was achieved as a result of surgical resection followed by a reduction in immunosuppression and low-dose rituximab-based chemotherapy, indicating that this therapeutic strategy may be safe and effective for the treatment of specific cases of localized and resectable PTLD.
\end{abstract}

\section{Introduction}

Post-transplant lymphoproliferative disorder (PTLD) is a rare, but critical complication that occurs following solid organ and hematopoietic stem cell transplantation (1). PTLD encompasses a heterogeneous group of disorders, ranging from benign self-limited lesions to aggressive widely disseminated disease. Generally, PTLD is considered to be an iatrogenic complication due to the intensive immunosuppressive treatment administered following transplantation (2). The overall incidence of PTLD in adult kidney transplantation surgery was between 1 and 3\% worldwide (3). However, early-onset PTLD ( $<1$ year from transplantation to presentation of PTLD) is closely associated with the Epstein-Barr virus (EBV) infection and exhibits a predilection for allograft localization (4), occasionally occuring at sites adjacent to the allograft. The current study describes a rare case of PTLD that presented as

Correspondence to: Dr Longkai Peng, Department of Urological Organ Transplantation, Second Xiangya Hospital, Central South University, 139 Renmin Road, Changsha, Hunan 41011, P.R. China E-mail: gylchygc@126.com

Key words: post-transplant lymphoproliferative disorder, Epstein-Barr virus, early-onset, kidney transplantation a tumor adjacent to the allograft within the first year following renal transplantation. The treatment strategy included surgical resection that was followed by a reduction in immunosuppression and low-dose rituximab-based chemotherapy. This study may lead to future improvements for the treatment of post-transplant lymphoproliferative disorder. Written informed consent was obtained from the patient.

\section{Case report}

In March 2012, a 42-year-old male exhibiting end-stage renal disease secondary to hypertension received a kidney transplant at the Second Xiangya Hospital of Central South University (Changsha, China). The donor was a 27-year-old male who had succumbed to cardiac failure caused by craniocerebral trauma. The human leukocyte antigen (HLA) type of the donor and the recipient were A11/A2-B58/B13-DR4/DR53, DR9/DR53 and A2/A2-B13/B61-DR15/DR51, DR9/DR53, respectively. The recipient was EBV seronegative, however, the donor's EBV serologic status was unknown, as EBV serologic status was not tested routinely at the time of donation. Furthermore, the donor and recipient were seronegative for cytomegalovirus, and the Hepatitis B and C viruses. There were no complications during surgical follow-up. The patient's post-transplant immunosuppressive regimen included: Intravenous methylprednisolone (dose during surgery, $0.5 \mathrm{~g}$; dose for the first three days following surgery, $0.5 \mathrm{~g} / \mathrm{day}$ ); followed by cyclosporine (CsA; initial dose, $6 \mathrm{mg} / \mathrm{kg} / \mathrm{day}$; trough concentration was adjusted to $220-250 \mathrm{ng} / \mathrm{ml} \mathrm{0-6} \mathrm{months} \mathrm{following} \mathrm{transplantation} \mathrm{and}$ 180-220 ng/ml over the next 6-12 months); oral mycophenolate mofetil (MMF; dose, $0.75 \mathrm{~g}$ per $12 \mathrm{~h}$; gradually reduced to $0.5 \mathrm{~g}$ per $12 \mathrm{~h}$ for long-term maintenance immunosuppression); and prednisolone (initial dose, $80 \mathrm{mg} /$ day; gradually reduced to $10 \mathrm{mg} /$ day over the next 6 months for long-term maintenance immunosuppression). Good renal allograft function was observed immediately following surgery. On the twelfth postoperative day, the patient exhibited a serum creatinine level of $133 \mu \mathrm{mol} / 1$ (normal range, $44-133 \mu \mathrm{mol} / \mathrm{l}$ ) and was discharged. The allograft function remained normal during the out-patient follow-up, however, seven months post-transplantation, the patient developed a fever, oliguria and an elevated serum creatinine level $(410.7 \mu \mathrm{mol} / \mathrm{l})$. Sonography 

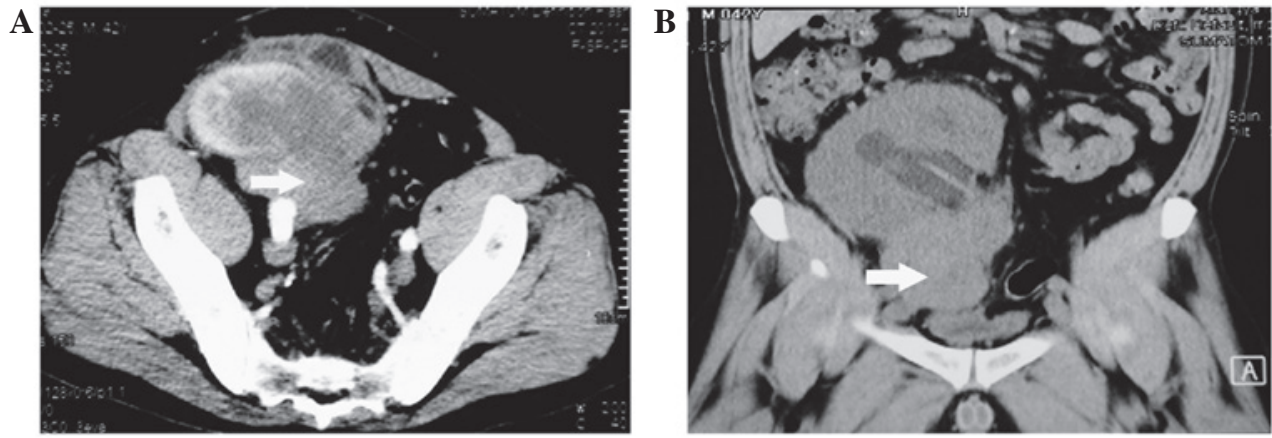

Figure 1. (A) Abdominal computed tomography showing a solid mass adjacent to the renal allograft. (B) Abdominal computed tomography with coronal multiplanar reformation showing a solid mass adjacent to the lower pole of the renal allograft and hydronephrosis. The arrow indicates the solid mass.
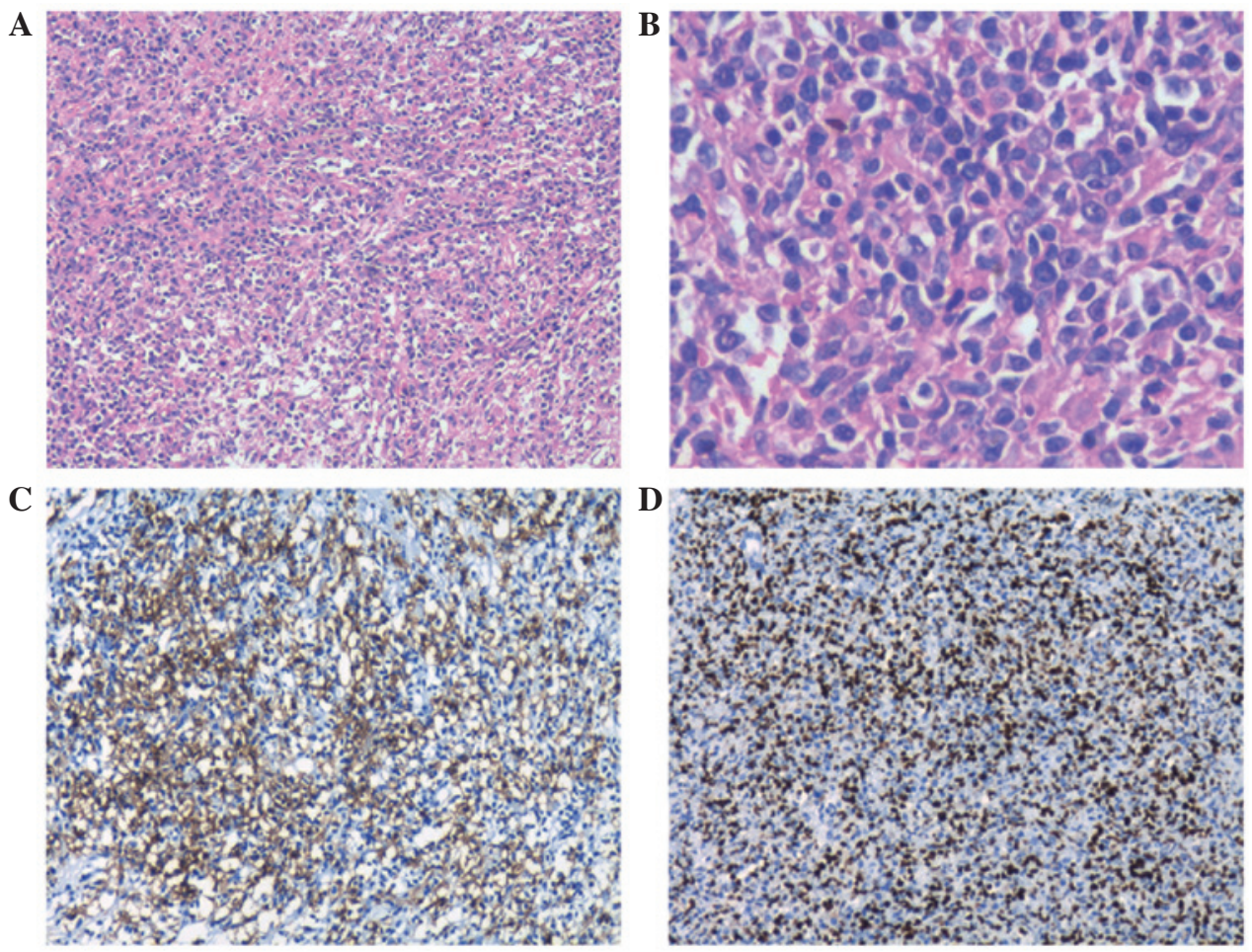

Figure 2. Histopathological images of the tumor. (A and B) Diffuse infiltration of lymphocytes, immunoblasts and plasma cells (stain, hematoxylin and eosin), Abundant lymphoid cells were positive for (C) cluster of differentiation 20 (staining, brown) and (D) Epstein-Barr virus-encoded RNA in situ hybridization (staining, brown). Magnification, (A, C and D) x100; (B) x400.

and computed tomography revealed a solid mass adjacent to the renal allograft (Fig. 1A) and computed tomography with coronal multiplanar reformation revealed a solid mass (size, $6 \times 4 \times 8 \mathrm{~cm}$ ) in the lower pole of the allograft and hydronephrosis (Fig. 1B). Percutaneous nephrostomy tubes were inserted, resulting in a decline in the serum creatinine level. Subsequently, surgical resection was performed and a tumor with a poorly defined margin, located adjacent and in close proximity to the lower pole of the allograft, was removed. Postoperatively, serum creatinine returned to within the normal range following treatment of the urinary obstruction. Intra- and postoperative histopathological assessments determined a diagnosis of polymorphic PTLD with positive stains for cluster of differentiation (CD)20, CD79a, CD3 and EBV-encoded RNA (Fig. 2). The proliferation index of Ki-67 was 40\%. Upon diagnosis, the blood EBV DNA level was $1.3 \times 10^{4}$ copies $/ \mathrm{ml}$, and the lactate dehydrogenase level (normal range, 135-215 U/l) and bone marrow biopsy were normal. Therefore, MMF therapy was discontinued and CsA was replaced with Rapamune (Wyeth Pharmaceuticals, Dallas, TX, USA) (trough concentration adjusted to 6-8 $\mathrm{ng} / \mathrm{ml}$ ) to reduce the level of immunosuppression. Furthermore, four cycles of adjuvant low-dose chemotherapy were administered, including rituximab $\left(300 \mathrm{mg} / \mathrm{m}^{2}\right)$, cyclophosphamide $\left(500 \mathrm{mg} / \mathrm{m}^{2}\right)$, vincristine $\left(1.2 \mathrm{mg} / \mathrm{m}^{2}\right)$ and prednisolone $\left(50 \mathrm{mg} / \mathrm{m}^{2}\right)$. The patient's blood was negative for EBV DNA following the first cycle of chemotherapy. During the 16-month follow-up after resection, the patient remained in remission, neither EBV viremia nor PTLD recurred and renal allograft function was preserved. Outpatient follow-up is ongoing to determine the long-term outcome of the treatment strategy. 


\section{Discussion}

PTLD is the second most commonly occurring malignancy in solid organ transplant recipients, worldwide. Approximately $20 \%$ of kidney transplant patients developed PTLD within the first year following surgery (5). Early-onset PTLD occurs more commonly in EBV seronegative recipients compared with EBV seropositive recipients and is characterized by an EBV in situ hybridization-positive, CD20-positive phenotype and allograft involvement (6). An immunosuppressed state and EBV infection are considered to be the two most important risk factors in PTLD development (2). In the majority of EBV-associated cases of PTLD, immunosuppression depresses the EBV-specific cellular immune response, which may promote uncontrolled EBV-infected lymphocyte proliferation, resulting in PTLD (7). The rare case described in the current report presented as a tumor adjacent to the lower pole of the renal allograft and developed into a urinary obstruction. To the best of our knowledge, few similar cases have been reported to date (8-12). In the present case, the risk factors for the development of PTLD included EBV seronegativity prior to transplantation, EBV infection post-transplantation, mismatching at the HLA-B locus and a high dose of CsA $(13,14)$.

No consensus on the optimal management of PTLD has been determined, however, a reduction in immunosuppression (RI) has been demonstrated to be an effective initial treatment modality for PTLD. In a recent analysis of 148 solid organ transplant-associated PTLD cases, Reshef et al (15) reported that the overall response rate for a RI alone was $45 \%$ and the three year overall survival rate was $55 \%$. Recent guidelines recommend commencing the reduction of immunosuppression therapy as soon as possible in all PTLD patients (16) and in specific cases of localized PTLD, surgical excision of isolated lesions or debulking of the tumor may be an effective component of first-line treatment. Reshef et al (15) identified that patients who underwent surgery and adjuvant RI exhibited a favorable outcome, with $27 \%$ patients relapsing at a median of five months. Rituximab is a monoclonal antibody against the B lymphocyte-specific CD20 antigen (17). Recent data indicates that immediate commencement of rituximab-based therapy followed by anthracycline-based chemotherapy (cyclophosphamide, doxorubicin, vincristine and prednisolone) may result in durable progressive-free survival in PTLD patients (18) and may reduce the risk of renal graft impairment following the reduction of immunosuppression (19). In the present case, surgical excision was performed for the diagnosis and resolution of the urinary obstruction. RI, followed by rituximab-based therapy combined with low-dose chemotherapy, for four months, the standard dose is as follows: rituximab $\left(375 \mathrm{mg} / \mathrm{m}^{2}\right.$, day 1$)$, cyclophosphamide $\left(400 \mathrm{mg} / \mathrm{m}^{2}\right.$, days $1-5)$, vincristine $\left(1.4 \mathrm{mg} / \mathrm{m}^{2}\right.$, day 1$)$ and prednisolone $\left(100 \mathrm{mg} / \mathrm{m}^{2}\right.$, days $\left.1-5\right)$ and was prescribed due to the aggressive nature of the disease. The therapeutic strategy administered to the patient in the present study resulted in complete remission with few manageable side-effects, including nausea/vomiting and leukopenia. Consistent with the present case, two previous studies reported that surgical intervention in combination with other therapies achieved durable remission in specific patients exhibiting localized PTLD $(20,21)$. The maintenance of immunosuppression remains a challenge in renal recipients who develop PTLD. The use of calcineurin inhibitors has been associated with an increased incidence of PTLD (22), however, treatment with rapamycin and its analogs has demonstrated immunosuppression and antiproliferative action. Previous studies demonstrated that rapamycin immunosuppressant therapy produced favorable effects in kidney transplant patients exhibiting PTLD $(23,24)$. Therefore, serolimus was introduced in the present case for maintenance immunosuppression. However, the use of rapamycin in PTLD patients remains controversial (25), thus, further studies are required to reach a consensus for the optimal management of PTLD.

In conclusion, the current report presents a rare case of PTLD that manifested as an obstructive uropathy within the first year following kidney transplantation. Surgical excision followed by a reduction in immunosuppression and low-dose rituximab-based chemotherapy may present as an effective and safe strategy for specific cases of localized and resectable PTLD.

\section{References}

1. Everly MJ, Bloom RD, Tsai DE and Trofe J: Posttransplant lymphoproliferative disorder. Ann Pharmacother 41: 1850-1858, 2007.

2. Dierickx D, Tousseyn T, De Wolf-Peeters C, Pirenne J and Verhoef G: Management of posttransplant lymphoproliferative disorders following solid organ transplant: an update. Leuk Lymphoma 52: 950-961, 2011.

3. Caillard S, Lelong C, Pessione F and Moulin B; French PTLD Working Group: Post-transplant lymphoproliferative disorders occurring after renal transplantation in adults: report of 230 cases from the French Registry. Am J Transplant 6: 2735-2742, 2006.

4. Khedmat $\mathrm{H}$ and Taheri S: Early onset post transplantation lymphoproliferative disorders: analysis of international data from 5 studies. Ann Transplant 14: 74-77, 2009.

5. Morton M, Coupes B, Roberts SA, et al: Epidemiology of posttransplantation lymphoproliferative disorder in adult renal transplant recipients. Transplantation 95: 470-478, 2013.

6. Ghobrial IM, Habermann TM, Macon WR, et al: Differences between early and late posttransplant lymphoproliferative disorders in solid organ transplant patients: are they two different diseases? Transplantation 79: 244-247, 2005.

7. Tsao L and Hsi ED: The clinicopathologic spectrum of posttransplantation lymphoproliferative disorders. Arch Pathol Lab Med 131: 1209-1218, 2007.

8. Kew CE II, Lopez-Ben R, Smith JK, et al: Posttransplant lymphoproliferative disorder localized near the allograft in renal transplantation. Transplantation 69: 809-814, 2000.

9. Palmer BF, Sagalowsky AI, McQuitty DA, Dawidson I, Vazquez MA and Lu CY: Lymphoproliferative disease presenting as obstructive uropathy after renal transplantation. J Urol 153: 392-394, 1995.

10. Khedmat $\mathrm{H}$ and Taheri S: Characteristics and prognosis of post-transplant lymphoproliferative disorders within renal allograft: Report from the PTLD. Int. Survey. Ann Transplant 15: 80-86, 2010.

11. Cosio FG, Nuovo M, Delgado L, et al: EBV kidney allograft infection: possible relationship with a peri-graft localization of PTLD. Am J Transplant 4: 116-123, 2004.

12. Caillard S, Porcher R, Provot F, et al: Post-transplantation lymphoproliferative disorder after kidney transplantation: report of a nationwide French registry and the development of a new prognostic score. J Clin Oncol 31: 1302-1309, 2013.

13. Quinlan SC, Pfeiffer RM, Morton LM and Engels EA: Risk factors for early-onset and late-onset post-transplant lymphoproliferative disorder in kidney recipients in the United States. Am J Hematol 86: 206-209, 2011.

14. Bakker NA, van Imhoff GW, Verschuuren EA, et al: HLA antigens and post renal transplant lymphoproliferative disease: HLA-B matching is critical. Transplantation 80: 595-599, 2005.

15. Reshef R, Vardhanabhuti S, Luskin MR, et al: Reduction of immunosuppression as initial therapy for posttransplantation lymphoproliferative disorder. Am J Transplant 11: 336-347, 2011. 
16. Parker A, Bowles K, Bradley JA, et al; Haemato-oncology Task Force of the British Committee for Standards in Haematology and British Transplantation Society: Management of post-transplant lymphoproliferative disorder in adult solid organ transplant recipients - BCSH and BTS Guidelines. Br J Haematol 149: 693-705, 2010

17. Svoboda J, Kotloff R and Tsai DE: Management of patients with post-transplant lymphoproliferative disorder: the role of rituximab. Transpl Int 19: 259-269, 2006.

18. Trappe R, Oertel S, Leblond V, et al; German PTLD Study Group; European PTLD Network: Sequential treatment with rituximab followed by CHOP chemotherapy in adult B-cell post-transplant lymphoproliferative disorder (PTLD): the prospective international multicentre phase 2 PTLD-1 trial. Lancet Oncol 13: 196-206, 2012.

19. Trappe R, Hinrichs C, Appel U, et al: Treatment of PTLD with rituximab and CHOP reduces the risk of renal graft impairment after reduction of immunosuppression. Am J Transplant 9: 2331-2337, 2009.

20. Foroncewicz B, Mucha K, Usiekniewicz J, et al: Posttransplant lymphoproliferative disorder of the lung in a renal transplant recipient treated successfully with surgery. Transplant Proc 38: 173-176, 2006.
21. Moudouni SM, Tligui M, Doublet JD, Haab F, Gattegno B and Thibault P: Lymphoproliferative disorder presenting as a tumor of the renal allograft. Int Urol Nephrol 38: 779-782, 2006.

22. André N, Roquelaure B and Conrath J: Molecular effects of cyclosporine and oncogenesis: a new model. Med Hypotheses 63: 647-652, 2004.

23. Pascual J: Post-transplant lymphoproliferative disorder - the potential of proliferation signal inhibitors. Nephrol Dial Transplant 22 (Suppl 1): i27-i35, 2007.

24. Alexandru S, Gonzalez E, Grande C, et al: Monotherapy rapamycin in renal transplant recipients with lymphoma successfully treated with rituximab. Transplant Proc 41: 2435-2437, 2009.

25. DiNardo CD and Tsai DE: Treatment advances in posttransplant lymphoproliferative disease. Curr Opin Hematol 17: 368-374 2010. 